\title{
Optimizing the branch number and branch length of radial drilling in high water cut low permeability reservoirs
}

\author{
Xiu-Kun Wang ${ }^{1} \cdot$ Chuan-Zhi Cui ${ }^{1}$
}

Received: 28 December 2014/ Accepted: 17 May 2015/Published online: 30 May 2015

(C) The Author(s) 2015. This article is published with open access at Springerlink.com

\begin{abstract}
Radial drilling, also called ultra-short horizontal well, is a new kind stimulation technology, which can be used both in new and old wells to improve the productivity and oil recovery effectively. For the low permeability reservoirs, it had been widely used in practice to effectively solve the unbalanced displacement problems for the waterflooded reservoirs. Applying the multi-layer equilibrium displacement principle, the model of optimizing the radial drilling branch number and length for the high water cut low permeability reservoirs is established, and the corresponding programs are also compiled. Using the reservoir numerical simulation technology, this model is proved to be valid and accurate. This optimization method has been applied in Bonan fifth reservoir of Shengli oilfield, which exhibits quite positive results: higher average production rate and lower average water cut of the radial drilled wells.
\end{abstract}

Keywords Radial drilling $\cdot$ High water cut $\cdot$ Low permeability $\cdot$ Equilibrium displacement

\section{List of symbols}

a Branch length

$f_{\text {we }}$ The water cut when the exit end's water saturation is $S_{\text {we }}$

$f^{\prime}{ }_{\text {we }}$ The water cut increasing rate when the exit end's water saturation is $S_{\text {we }}$

$G \quad$ Starting pressure gradient

$h \quad$ The sickness of the layer

Xiu-Kun Wang

xkwang1990@gmail.com

1 China University of Petroleum (East China), Qingdao, China $h_{\mathrm{o}} \quad$ The distance between the branch well and the bottom of the layer

$k \quad$ Horizontal permeability

$k_{\text {ro }} \quad$ Relative permeability of oil

$k_{\mathrm{rw}} \quad$ Relative permeability of water

$n \quad$ Branch number

$k_{\mathrm{z}} \quad$ Vertical permeability

$p_{\mathrm{f}} \quad$ Pressure of the fracture

$\mathrm{PVi}$ The $i$ th layer's pore volume

$\Delta p \quad$ Pressure drop

$Q \quad$ Production rate

$\mathrm{R}_{\mathrm{e}} \quad$ Drainage radius

$R_{\mathrm{ij}} \quad$ The $i$ th layer's flow resistance when the water saturation is between $S_{\text {wei }}+j \Delta S_{\mathrm{w}}$ and $S_{\text {wei }}+$ $(j+1) \Delta S_{\mathrm{w}}$

$r_{\mathrm{w}} \quad$ Well radius

$r_{\mathrm{wh}} \quad$ Radius of the lateral well

$S_{\text {we }} \quad$ Water saturation of the exit end

$\overline{S_{\mathrm{we}}} \quad$ The average water saturation when the exit end's water saturation is $S_{\text {we }}$

$\mu \quad$ Viscosity of the fluid

$\mu_{\mathrm{o}} \quad$ Oil viscosity

$\mu_{\mathrm{w}} \quad$ Water viscosity

\section{Introduction}

Radial drilling is already not a new technique in the petroleum engineering area. According to the state of art (Marbun et al. 2011), radial drilling is implemented through a special high pressure tube to form the water jet to penetrate and drill several lateral boreholes in one or several layers, which is an effective way to increase the drainage area and improve the oil recovery. Dickinson 
et al. (1989) first introduced the radial drilling system and predicted the promising expectation of this drilling system. Dickinson et al. (1992) presented two methods of combining water jet drilling and coiled tubing, and discussed their advantages and weaknesses in the practice. Yang et al. (2006) introduced the technique status in China and its application situation in Liaohe oilfield. Bruni et al. (2007) exhibited the Radial drilling technique in Argentina. Ursegov et al. (2008) presented the first results of cyclic steam stimulations of vertical wells with radial horizontal bores in heavy oil carbonates, and the results validated the wide usability of radial drilling technique. And AbdelGhany et al. (2011) stated the first radial drilling well conditions in Egypt. As for all of the researches above, most of them are just focused on the application status or the techniques of the radial drilling system, but almost no research was carried out to probe how to optimize the radial drilling branch length and branch number in terms of reservoir engineering respect, which is quite important for the multi-layer water drive reservoirs.

In the low permeability reservoirs, several layers are produced together, due to the differences in the rock properties, the flow abilities of different layers are quite various, which lead to the conflicts of multi-layer, and unbalanced displacement performance. In the practice, engineers tried to solve this problem using the radial drilling technique in the poor property layers to drill several boreholes in order to improve their flow ability, which is proved to be really effective. But, there is still no theoretical basis to guide the engineers to decide how long the lateral wells should be drilled and how many number of the lateral branch bores should be implemented. So, herein according to the equilibrium displacement principle, applying the equivalent flow resistance method, the model of optimizing the radial drilling branch number and length in the high water cut low permeability reservoirs is established.

In this paper, we first present the methodology in order to clarify the basic thread of this model. Accordingly, we analyze the productivity of a radial drilling well, and different kinds of flow resistances are presented in this section. In the third section, the equilibrium displacement principle is discussed, and the calculation steps are presented. Last, using the reservoir numerical technology, this method is verified to be valid and accurate.

\section{Methodology}

In order to obtain equilibrium displacement, the poor property layers (lower permeability, thinner thickness, etc.) are implemented with radial drilling. Then flow ability of those layers with the radial boreholes is quite improved, but we do not expect it to be overbalanced, so, the multilayer equilibrium displacement principle is introduced as the optimization basis. The model is established based on the constant pressure difference between the production and injection wells. During the optimization process, the principle of analogy of water and electricity is applied, so the flow resistances are integrant variables that we need to valuate.

First, the time spent for the layer without the radial boreholes to get certain water saturation can be obtained, and given the branch number and branch length of the radial drilling wells, the time spent for the layer with radial boreholes to get the same water saturation is also calculated. Then, try to narrow the time difference by adjusting the values of the branch length and branch number, and the ultimate results are the optimal branch length and branch number that are needed to be implemented, aiming at the multi-layer equilibrium displacement.

\section{Flow resistance computation}

Before analyzing the oil-water two phases flow, first, we need to consider the one-phase flow condition. After radial drilling, there are two ways for the formation fluid to flow into the vertical wellbore: (1) formation fluid directly flows into the vertical wellbore; (2) the formation fluid flows through the lateral branch wells then flows into the vertical wellbore (as shown in Fig. 1).

\section{Flow resistance of the formation fluid directly flowing into the vertical wellbore}

This kind of flow pattern is thought to be radial flow. Due to the low permeability of this reservoir, considering the starting pressure gradient, the flow equation is written as

$v=\frac{k}{\mu}\left(\frac{\partial p}{\partial r}-G\right)$.

Integral Eq. 1 to get

$Q=\frac{2 \pi k h}{\mu \ln R_{\mathrm{e}} / r_{\mathrm{w}}}\left(\Delta p-G R_{\mathrm{e}}\right)$.

According to the analogy of water and electricity, the corresponding flow resistance, $R_{\mathrm{v}}$, is

$R_{v}=\frac{\mu}{2 \pi k h} \ln \frac{R_{\mathrm{e}}}{r_{\mathrm{w}}}$.

Flow resistance of the formation fluid flowing through the lateral branch wellbores then to the vertical wellbore

This kind of flow pattern can be separated into two flow processes (Zhaoxin 2001): the outer flow process and the inner flow process. The outer flow process is thought to be 
Fig. 1 Two ways that formation fluid flows into the vertical wellbore
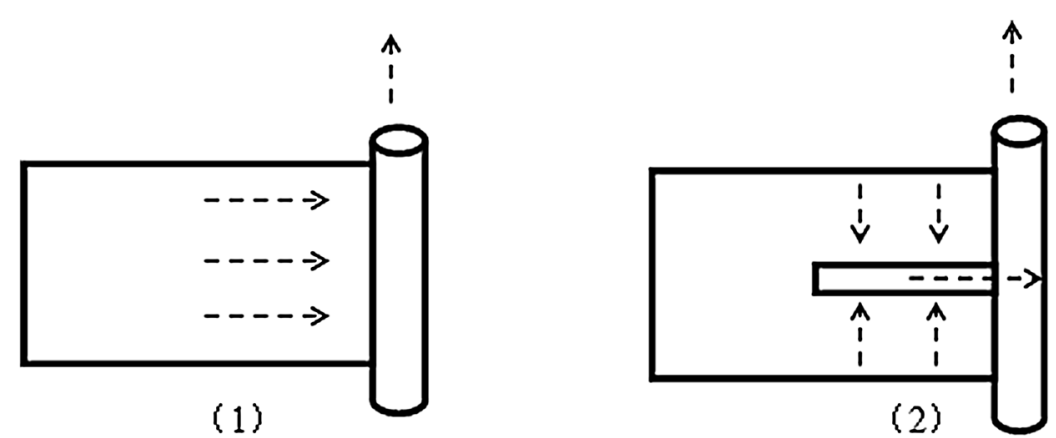

the formation fluid flowing into the multiple fractured well, and the inner process to be a radial flow in the vertical plane with paralleled boundaries.

Outer flow process: flowing into the multiple fractured well (shown in Fig. 2).

Using the conformal transformation method, choose the transformation function as

$\left(\frac{z}{a}\right)^{n / 2}=\operatorname{ch} \varsigma$.

Spreading the imaginary part and the real part of the above equation, we can get

$\left(\frac{R}{a}\right)^{n / 2} e^{i n \theta / 2}=\left(\frac{R}{a}\right)^{n / 2}\left(\cos \frac{n \theta}{2}+i \sin \frac{n \theta}{2}\right)$

$\operatorname{ch}(\xi+i \eta)=\operatorname{ch} \xi \cos \eta+i \operatorname{sh} \xi \sin \eta$

That is

$\left(\frac{R}{a}\right)^{n / 2} \cos \frac{n \theta}{2}=\operatorname{ch} \xi \cos \eta$
$\left(\frac{R}{a}\right)^{n / 2} \sin \frac{n \theta}{2}=\operatorname{sh} \xi \sin \eta$.

By eliminating $\eta$, the Equipotential line equation is obtained

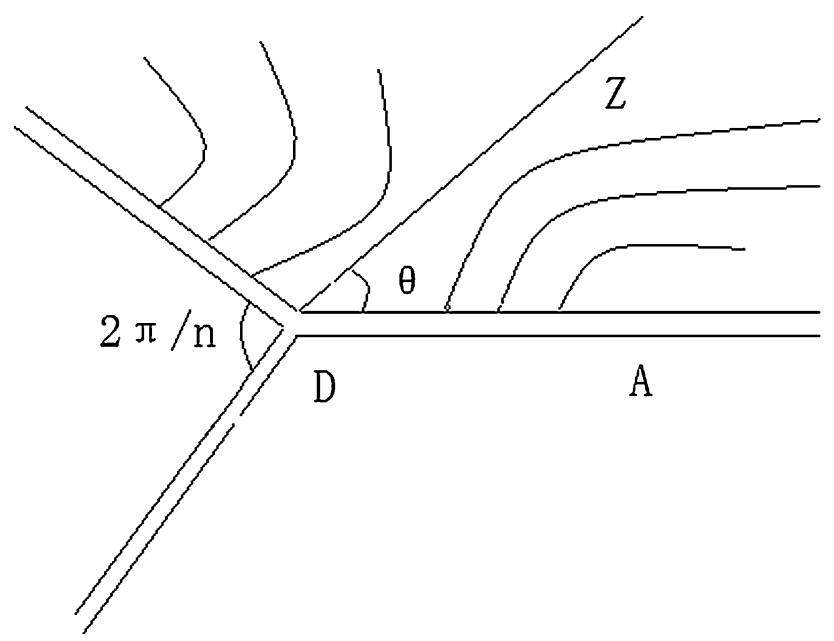

Fig. 2 Flow into the multiple fractured well
$\frac{\left(\frac{R}{a}\right)^{n / 2} \cos ^{2} \frac{n \theta}{2}}{\operatorname{ch}^{2} \xi}+\frac{\cos \eta\left(\frac{R}{a}\right)^{n / 2} \sin ^{2} \frac{n \theta}{2}}{\operatorname{sh}^{2} \xi}=1$.

When $\xi$ is big enough, $\operatorname{ch}^{2} \xi \approx \operatorname{sh}^{2} \xi=\frac{e^{2 \xi}}{4}$, then

$\left(\frac{R}{a}\right)^{n / 2}=\frac{e^{2 \xi}}{4}$.

Then

$R=a\left(\frac{e^{2 \xi}}{4}\right)^{1 / n}, \quad \xi=\frac{n}{2} \ln \left(4^{\frac{1}{n}} \frac{R}{a}\right)$.

Applying the superposition principle, we can get

$\Phi_{\mathrm{e}}-\Phi_{\mathrm{w}}=\frac{q}{2 \pi} \xi=\frac{q}{2 \pi} \frac{n}{2} \ln \left(\frac{4^{\frac{1}{n}} R}{a}\right)$.

The flow rate of the multiple fractured well $\mathrm{Q}$ is $\mathrm{nq} / 2$, so, the final productivity equation is developed as

$\Phi_{\mathrm{e}}-\Phi_{\mathrm{w}}=\frac{Q}{2 \pi} \ln \left(\frac{4^{\frac{1}{n}} R}{a}\right)$.

So

$\frac{2 \pi k h}{\mu} \frac{\left(p_{i}-p_{f}-G R_{\mathrm{e}}\right)}{Q}=\ln \frac{4^{\frac{1}{n}} R_{\mathrm{e}}}{a}$.

Inner flow process: radial flow in the vertical plane with paralleled boundaries (shown in Fig. 3).

Considering that the lateral permeability and the vertical permeability are different, and the ratio is

$\beta=\sqrt{\frac{k_{\mathrm{h}}}{k_{\mathrm{v}}}}$.

So, the equivalent radius is introduced

$r_{\mathrm{wh}}^{\prime}=\frac{1+\beta}{2} r_{\mathrm{wh}}$.

Applying the image theory, the flow pattern can be converted to a line of infinite producers. Therefore, the productivity equation is obtained as

$\Delta \Phi=\frac{q}{2 \pi} \ln \left[\frac{\pi y_{0}}{d}+\ln \frac{d / \sin (\pi a / d)}{2 \pi r_{\mathrm{w}}}\right]$. 


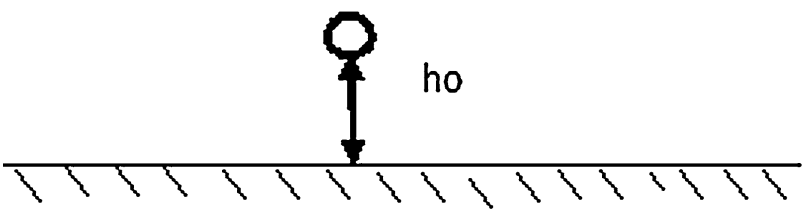

Fig. 3 Radial flow in the vertical plane with paralleled boundaries

Equation 15 can be rewritten as

$p_{\mathrm{f}}-p_{\mathrm{w}}=\frac{\mu Q}{2 \pi k h} \frac{\beta h}{n a} \ln \frac{\beta h / \sin \left(\pi h_{o} / h\right)}{\pi(1+\beta) r_{\mathrm{wh}}}$.

Combine the Eqs. 12 and 16, then

$\Delta p_{\mathrm{w}}-G R_{\mathrm{e}}=\frac{Q \mu}{2 \pi k h}\left[\ln \frac{4^{\frac{1}{n}} R_{\mathrm{e}}}{a}+\frac{\beta h}{n a} \ln \frac{\beta h / \sin \left(\pi h_{o} / h\right)}{\pi(1+\beta) r_{\mathrm{wh}}}\right]$.

According to the analogy of water and electricity, the corresponding flow resistance, $R_{\mathrm{h}}$, is

$R_{\mathrm{h}}=\frac{\mu}{2 \pi k h}\left[\ln \frac{4^{\frac{1}{n}} R_{\mathrm{e}}}{a} \frac{\beta h}{n a} \ln \frac{\beta h / \sin \left(\pi h_{o} / h\right)}{\pi(1+\beta) r_{\mathrm{wh}}}\right]$.

Flow resistance of the layer with radial boreholes in the low permeability reservoirs

The above two ways of flowing into the vertical wellbore happened simultaneously. So this can be considered to be the parallel connection relationship of the flow resistances $R_{\mathrm{h}}$ and $R_{\mathrm{v}}$. According to the analogy of water and electricity, the flow resistance of the layer implemented by the radial drilling, is obtained.

$R=\frac{\mu}{2 \pi k h} \frac{\left[\ln \frac{4 \frac{1}{n} R_{\mathrm{e}}}{a}+\frac{\beta h}{n a} \ln \frac{\beta h / \sin \left(\pi h_{o} / h\right)}{\pi(1+\beta) r_{\mathrm{wh}}}\right] \ln \frac{R_{\mathrm{e}}}{r_{\mathrm{w}}}}{\ln \frac{4 \frac{1}{n} R_{\mathrm{e}}^{2}}{a r_{\mathrm{w}}}+\frac{\beta h}{n a} \ln \frac{\beta h / \sin \left(\pi h_{o} / h\right)}{\pi(1+\beta) r_{\mathrm{wh}}}}$

Oil \& water flow resistance of the layer with radial boreholes in the low permeability reservoirs

Water saturation along the whole layer is distributed unequally and time dependently during the production of the reservoir, so in order to get the layer's flow resistance, we substitute the flow resistance of the average water saturation for the whole layer's flow resistance. When the water saturation of the exit end is $S_{\text {wej }}$, the corresponding average water saturation of the layer is $\overline{S_{\text {wej }}}$. According to Eq. 19, the oil phase flow resistance is proposed as
$R_{\mathrm{oj}}=\frac{\mu_{\mathrm{o}}}{2 \pi k k_{\mathrm{ro}}\left(\overline{S_{\mathrm{wej}}}\right) h} \frac{\left[\ln \frac{4 \frac{1}{n_{\mathrm{e}}}}{a}+\frac{\beta h}{n a} \ln \frac{\beta h / \sin \left(\pi h_{o} / h\right)}{\pi(1+\beta) r_{\mathrm{wh}}}\right] \ln \frac{R_{\mathrm{e}}}{r_{\mathrm{w}}}}{\ln \frac{4 \frac{1}{n} R_{\mathrm{e}}^{2}}{a r_{\mathrm{w}}}+\frac{\beta h}{n a} \ln \frac{\beta h / \sin \left(\pi h_{o} / h\right)}{\pi(1+\beta) r_{\mathrm{wh}}}}$.

Similarly the water phase flow resistance is developed as

$R_{\mathrm{wj}}=\frac{\mu_{\mathrm{w}}}{2 \pi k k_{\mathrm{rw}}\left(\overline{S_{\mathrm{wej}}}\right) h} \frac{\left[\ln \frac{4^{\frac{1}{n}} R_{\mathrm{e}}}{a}+\frac{\beta h}{n a} \ln \frac{\beta h / \sin \left(\pi h_{o} / h\right)}{\pi(1+\beta) r_{\mathrm{wh}}}\right] \ln \frac{R_{\mathrm{e}}}{r_{\mathrm{w}}}}{\ln \frac{4^{\frac{1}{n}} R_{\mathrm{e}}^{2}}{a r_{\mathrm{w}}}+\frac{\beta h}{n a} \ln \frac{\beta h / \sin \left(\pi h_{o} / h\right)}{\pi(1+\beta) r_{\mathrm{wh}}}}$.

Because of the parallel connection relationship of them (Jiali 2003),

$\frac{1}{R_{\mathrm{ij}}}=\frac{1}{R_{\mathrm{oj}}}+\frac{1}{R_{\mathrm{wj}}}$.

We can get the layer's whole flow resistance by Eqs. 20, 21 , and 22.

In the same way, as for the conventional layer without the radial drilling boreholes, the oil phase flow resistance is

$R_{\mathrm{oj}}=\frac{\mu_{o}}{2 \pi k k_{\mathrm{ro}}\left(\overline{S_{\text {wej }}}\right) h} \ln \frac{R_{\mathrm{e}}}{r_{\mathrm{w}}}$.

The water phase flow resistance is

$R_{\mathrm{wj}}=\frac{\mu_{\mathrm{w}}}{2 \pi k k_{\mathrm{rw}}\left(\overline{S_{\mathrm{wej}}}\right) h} \ln \frac{R_{\mathrm{e}}}{r_{\mathrm{w}}}$.

Similarly, according to the parallel relationship, the whole layer's resistance can be obtained by Eqs. 23, 24, and 22.

\section{Optimization model}

As for equilibrium displacement, a lot of researchers had given some standards (Chuanzhi et al. 2012). In a simple way, for the multi-layer reservoirs, equilibrium displacement refers to the equal use of each layer during waterflooding process. In this paper, the equilibrium displacement is defined that in the end, the water cut of each layer reaches $98 \%$ in the same time. To be simple, choose the bilayer model as the research objective. Supposing that the first layer's properties are poor and this layer requires the implementation of radial drilling. The conductivity of the lateral branch wellbores is infinite, i.e., the pressure in the wellbore is the same. The flow pattern of each layer is the radial flow; the detailed model is shown in Fig. 4.

\section{Derivation of the basic theory of equilibrium displacement}

Using the production data, each layer's average water saturation ${\overline{S_{\mathrm{we}}}}^{*}$ can be calculated. According to the Welge equation, 


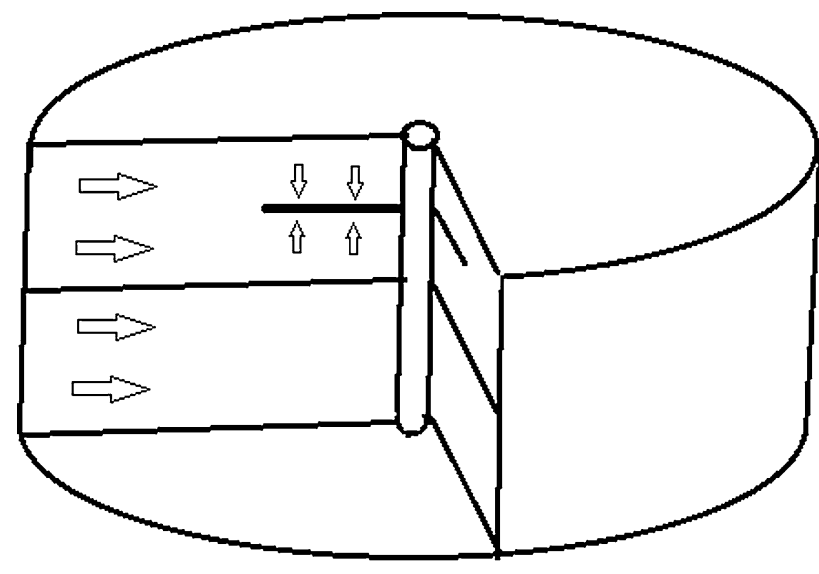

Fig. 4 The basic optimization model

$S_{\mathrm{we}}=\overline{S_{\mathrm{we}}}-\frac{1-f_{\mathrm{we}}}{f_{\mathrm{we}}^{\prime}}$.

Equation 28 can be solved using the iterative way. First, given a $S_{\mathrm{we}}$, then a corresponding $\overline{S_{\mathrm{we}}}$ can be obtained. If its difference with ${\overline{S_{\mathrm{we}}}}^{*}$ is quite big, try to change the value of $S_{\text {we }}$, until the obtained $\overline{S_{\text {we }}}$ is within the tolerated error. Therefore, through this method, the $i$ th layer's beginning water saturation $S_{\text {wei }}$ can be obtained.

Supposing after radial drilling measures, keeping the production pressure drop $\Delta p$ unchanged, the reservoir is continued to be produced for $\Delta t$ time, then equilibrium displacement is achieved and the water cut of all the layers now is $98 \%$, and all the layers' corresponding water saturation of

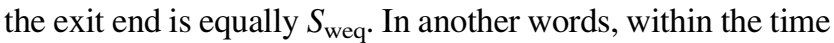
of $\Delta t$, each layer's water saturation of the exit end, increases from $S_{\text {wei }}$ to $S_{\text {weq }}$. Because the corresponding liquid production rate $q_{\mathrm{i}}$ is always changing, considering within a slight variation range $\Delta S_{\mathrm{w}}$, the liquid production rate $q_{\mathrm{i}}$ is thought to be constant. Hence, as for the ith layer, the variation of the water saturation from $S_{\text {wei }}+j \Delta S_{\mathrm{w}}$ to $S_{\text {wei }}+(j+1) \Delta S_{\mathrm{w}}$, the corresponding liquid production rate $q_{\mathrm{ij}}$ is

$q_{\mathrm{ij}}=\frac{\Delta p-G R_{e}}{R_{\mathrm{ij}}}$,

where $R_{\mathrm{ij}}$ can be obtained from Eq. 22, whether the layer is with or without the radial boreholes.

According to the Welge equation, the cumulated liquid production $\Delta W_{\mathrm{ij}}$ is

$$
\begin{aligned}
\Delta W_{\mathrm{ij}} & =\frac{P V i}{f_{\mathrm{w}}^{\prime}\left(S_{\mathrm{wei}}+(j+1) \Delta S_{\mathrm{w}}\right)}-\frac{P V i}{f_{\mathrm{w}}^{\prime}\left(S_{\mathrm{wei}}+j \Delta S_{\mathrm{w}}\right)} \\
& =\frac{\pi R_{\mathrm{e}}^{2} h_{\mathrm{i}}}{f_{\mathrm{w}}^{\prime}\left(S_{\mathrm{wei}}+(j+1) \Delta S_{\mathrm{w}}\right)}-\frac{\pi R_{\mathrm{e}}^{2} h_{\mathrm{i}}}{f_{\mathrm{w}}^{\prime}\left(S_{\mathrm{wei}}+j \Delta S_{\mathrm{w}}\right)} .
\end{aligned}
$$

So, the spent time $\Delta t_{\mathrm{ij}}$ is

$$
\Delta t_{\mathrm{ij}}=\frac{\Delta W_{\mathrm{ij}}}{q_{\mathrm{ij}}} .
$$

Hence, the cumulated time as for ith layer's water saturation of the exit end increasing from $S_{\text {wei }}$ to $S_{\text {weq }}$ is

$\Delta t_{\mathrm{i}}=\sum \Delta t_{\mathrm{ij}}$.

Due to the equilibrium displacement, hence,

$\Delta t_{\mathrm{i}}=\Delta t$.

\section{Specific calculation steps}

First, compute the time spent on the second layer, where the radial drilling is not implemented. Using the properties and the production data, the water saturation of the exit end $S_{\text {we2 }}$ is obtained. Then, applying Eqs. 20-22 and 26-29, the time spent $\Delta t_{2}$ for the water saturation of the exit end from $S_{\text {we2 }}$ to $S_{\text {weq }}$ is calculated.

As for the first layer that requires the radial drilling treatment, likewise, first, the water saturation of the exit end $S_{\text {we1 }}$ is obtained. Then, given the values of branch length $a$ and the branch number $n$, applying Eq. 22-29, the time spent $\Delta t_{1}$ for the water saturation of the exit end from $S_{\text {we } 1}$ to $S_{\text {weq }}$ is calculated. If the difference between $\Delta t_{1}$ and $\Delta t_{2}$ is not negligible, try to change the values of branch length $a$ and branch number $n$ to recalculate the time spent $\Delta t_{1}$, until the difference is reduced to the tolerant error. Then, the results are the optimal radial drilling branch number $n$ and branch length $a$ values that are used to realize the equilibrium displacement in the high water cut stage of the low permeability reservoirs.

This model can easily be carried out by the corresponding program, no detail is needed to be described.

\section{Model verification}

Supposing there is a group of wells in a low permeability reservoir, which consists of two layers, owning a same porosity, i.e., $20 \%$. The upper layer thickness is $5 \mathrm{~m}$, and the lower layer is $10 \mathrm{~m}$. And their permeabilities are $10 \times 10^{-3}$ and $30 \times 10^{-3} \mu \mathrm{m}^{2}$, respectively. The well spacing is $150 \mathrm{~m}$. After a period time of production, all the two layers have already broken through, and the corresponding current recoveries are 12.53 and $21.90 \%$, respectively.

According to the model herein, the optimal results for the multi-layer equilibrium displacement can be computed. That is, the radial branch number $n=2$, and the branch length $a=23 \mathrm{~m}$.

As for the above issue, apply the reservoir numerical simulation technology, and split the 21 layers vertically, and the middle layer is the impermeable layer to cut off the fluid connection between the upper and lower layers. The reservoir is produced for another 15 years in two different 
ways: one implemented the radial drilling in the upper layer, the other not.

The numerical results show that, as for the one that unimplemented the radial drilling, the recoveries of two layers are 24.30 and $33.76 \%$, respectively. And the one that implemented the radial drilling, yields the recoveries 32.62 and $33.29 \%$, respectively (shown in Fig. 5), nearly equal. Therefore, the conflicts of the layers are effectively solved.

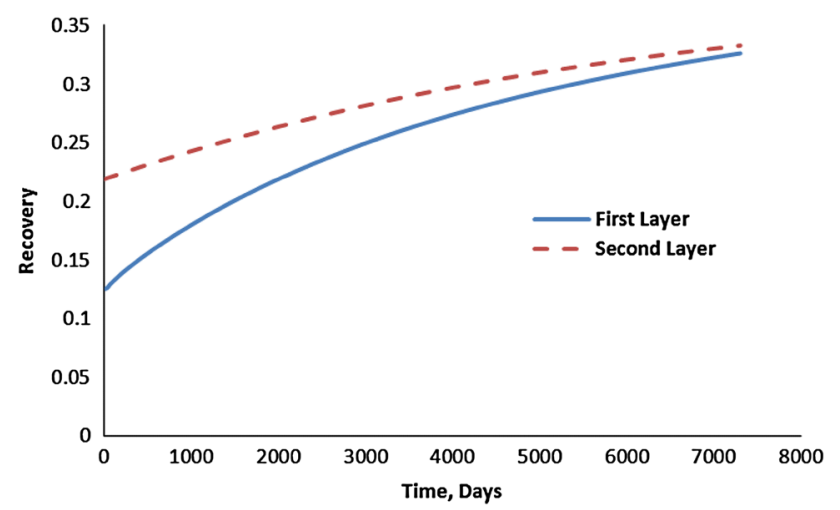

Fig. 5 The numerical simulation results of the two layers after radial drilling

\section{Field application}

Bonan fifth reservoir is located within Shengli oilfield of Shandong provence of China, which is believed to be a typical low permeability reservoir. The reservoir ranges from $S_{3} 1$ to $S_{3} 5$ formations, and the reserve is about $1759 \times 10^{4} \mathrm{t}$. The average permeability is about $20-30 \times 10^{-3} \mu^{2}$, and the reservoir began to inject water in 1978. Comparing with other low permeability reservoirs, Bonan fifth reservoir has its own characteristics: the water injection volumes are large, the water cut is high, and the oil recovery is low. All of those are mainly caused by the different properties of the multi-layers. So, radial drilling techniques are introduced in this reservoir in December, 2012.

Using the optimization methods in this paper, the radial drilling branch number and branch length of each layer is determined in order to realize the multi-layer equilibrium displacement. So far, eight wells have been implemented with radial drilling techniques, and the average oil production rate increases from 2.5 to $9.7 \mathrm{t} / \mathrm{d}$; the average water cut decreases from 75.3 to $63.8 \%$, and the accumulative enhanced oil is about $1.82 \times 10^{4} \mathrm{t}$. The production rate and production profile of well B5-36 are shown in Figs. 6 and 7.
Fig. 6 Production rate of well B5-36

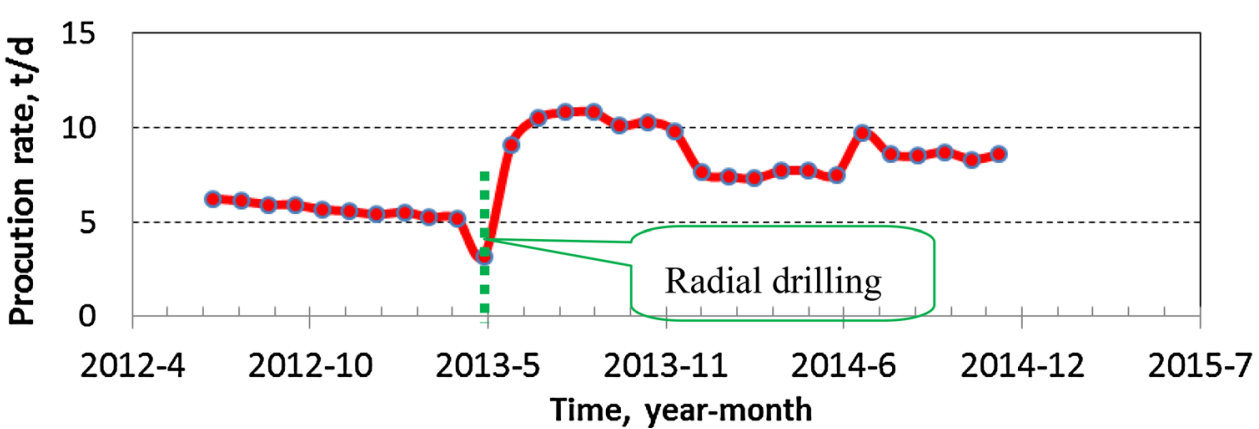

Fig. 7 Production profile change of well B5-36 before (a) and after (b) radial drilling

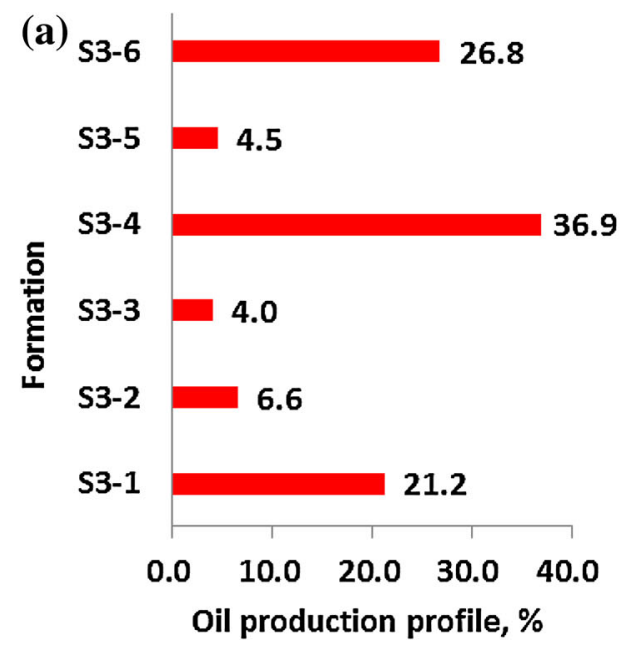




\section{Conclusions}

Based on the multi-layer equilibrium displacement principle, considering the starting pressure gradient, applying the equivalent flow resistance method, the model for optimizing the radial drilling branch number and length for the high water cut low permeability reservoirs is established. And the corresponding programs are developed.

The optimization method is used in a synthetic model, using the reservoir simulation technology; the results of the simulation proved the validation and accuracy. This optimization method has been applied in Bonan fifth reservoir of Shengli oilfield, which exhibits quite positive results: higher average production rate and lower average water cut of the radial drilled wells. Hence, the radial drilling technique is suggested to be a great tool to solve the conflicts of layers for the development of the multilayered reservoirs.

Acknowledgments This work was supported by Program for Changjiang Scholars and Innovative Research Team in University (IRT1294) and China National Science and Technology Major Projects (Topic name: The technology to improve oil recovery at high water cut period in Shengli oilfield, Topic number: 2011ZX05011-02).

Open Access This article is distributed under the terms of the Creative Commons Attribution 4.0 International License (http:// creativecommons.org/licenses/by/4.0/), which permits unrestricted use, distribution, and reproduction in any medium, provided you give appropriate credit to the original author(s) and the source, provide a link to the Creative Commons license, and indicate if changes were made.

\section{References}

Abdel-Ghany MA, Siso S, Hassan AM, Pierpaolo P, Roberto C (2011) New technology application. In: Radial drilling petrobel, first well in Egypt, presented at the 10th offshore mediterranean conference and exhibition (OMC) in Ravenna, Italy

Bruni M, Biasotti J, Salomone G (2007). Radial drilling in Argentina. In: Latin American \& Caribbean Petroleum Engineering Conference

Chuanzhi C, Hua J, Jiehong D et al (2012) Reasonable injection rate allocation method of separate-layer water injection wells based on interlayer equilibrium displacement. PGRE 19(5):94-96

Dickinson W, Anderson RR, Dickinson RW (1989) The ultrashortradius radial system. SPE Drilling Eng 4(3):247-254

Dickinson W, Dickinson R, Herrera A, Dykstra H, Nees J (1992) Slim hole multiple radials drilled with coiled tubing. In: SPE Latin America Petroleum Engineering Conference

Jiali Ge (2003) Modern reservoir flow mechanics principle. Petroleum Industry Press, Beijing

Marbun B, Zulkhifly S, Arliyando L, Putra SK (2011). Review of ultrashort-radius radial system (URRS). In: International Petroleum Technology Conference

Ursegov S, Bazylev A, Taraskin E (2008) First results of cyclic steam stimulations of vertical wells with radial horizontal bores in heavy oil carbonates (Russian). In: SPE Russian Oil and Gas Technical Conference and Exhibition

Yang Y, Yang H, Wang R, Ma K, Zhou W, Ma Q, Xu X (2006) Application of ultra-short radius radial wells drilling technology in wei-5 well. ODPT 28(2):11-14

Zhaoxin L (2001) Oil and gas underground flow mechanics. University of Petroleum Press, Dongying, pp 36-46 